\title{
Evaluación pesquera de especies de interés cultural y económico en el sistema lagunar de Karataska
}

Fisheries assessment of species of cultural and economic interest in the Karataska lagoon system

DOI 10.5377/rct.v0i24.7878

\author{
Nhering Daniel Ortiz Lobo \\ José Luis Andrade Campos ${ }^{2}$ \\ Ester Agar López Irías ${ }^{3}$ \\ Alexis Irías Coello ${ }^{4}$
}

\section{RESUMEN}

El plan de manejo pesquero para el Sistema Lagunar de Karataska en la Moskitia hondureña registró 80 especies distribuidas entre peces, moluscos y crustáceos de los cuales cerca del $40 \%$ de estas especies son de interés económico y cultural. Las comunidades indígenas miskitas hondureñas viven principalmente de la pesca artesanal tanto para el autoconsumo como para la venta a pequeña escala de robalo y otras especies. Con el objetivo de alcanzar el manejo sostenible de las pesquerías artesanales del robalo (Centropomus undecimalis, Centropomus pectinatus y Centropomus parallelus) y la kawacha (Eugerres plumieri) en la laguna de Karataska, el Proyecto Moskitia de PNUD/GEF con la asesoría científica de la UNAH, realizó una evaluación de las principales especies ícticas de interés cultural y económico del Sistema Lagunar de Karataska. Esta evaluación comprendió la aplicación del modelo analítico del enfoque cuantitativo en el periodo 2012-2014. La estimación de la mortalidad total (Z) fue de 1.78 para Centropomus undecimalis y 1.67 para Eugerres plumieri, lo que indica que ambas especies se encuentran en fases de explotación intensa, el análisis de cohorte de Jones muestra de forma muy preliminar que el rendimiento máximo sostenible (RMS) para Centropomus undecimalis es de 69,193.17 toneladas y para Eugerres plumieri es de 17,8604.59 toneladas. De esta manera el Proyecto Moskitia de PNUD/GEF y la UNAH aportan conocimientos científicos, con el fin de brindar herramientas de manejo a los pobladores miskitos para el ordenamiento de sus recursos pesqueros.

\footnotetext{
${ }^{1}$ Licenciado en Biología, Proyecto Moskitia / PNUD- GEF. Correo electrónico: nhering.lobo@gmail.com

${ }^{2}$ Lic. En Biología, Proyecto Moskitia / PNUD- GEF. Correo electrónico: joseluisandradecampos@ gmail. com

${ }^{3}$ Profesora universitaria, Escuela de Biología, Facultad de Ciencias, UNAH. Correo electrónico: ester. lopez@unah.edu.hn

${ }^{4}$ Licenciado en Biología, Proyecto Moskitia / PNUD- GEF. Correo electrónico: alexis.irias@undp.org
} 
Palabras clave: Sistema lagunar, pesca artesanal, manejo sostenible, recursos pesqueros, explotación mortalidad total, rendimiento máximo sostenible.

\section{ABSTRACT}

The fishing management plan for the Karataska Lagoon System in the Honduran Moskitia recorded 80 species distributed among fish, molluscs and crustaceans of which about $40 \%$ of these species are of economic and cultural interest. The miskitian Honduran indigenous communities sustain themselves mainly from the artisan fishing used for self-consumption or small-scale commerce of snook and other species. With the objective of achieving the sustainable management of artisanal fisheries of snook (Centropomus undecimalis, Centropomus pectinatus and Centropomus parallelus) and "kawacha" (Eugerres plumieri) in the Karataska lagoon, the Moskitia Project of PNUD/GEF with the scientific consultant's office of the UNAH, evaluated the main ichthyological species with cultural and economic interest in the Karataska Lagoon System. The evaluation included an analytical model for the quantitative approach between the years 2012-2014. The estimation of total mortality (z) was of 1,78 for Centropomus undecimalis and 1,67 for Eugerres plumieri, which indicates that both species are in intense exploitation phases, the analysis of cohort of Jones shows, in a very preliminary sample, that the peak efficiency sustainable (PES) for Centropomus undecimalis is of 69,193.17 tons and for Eugerres plumieri is of 17,8604.59 tons. With our results, Moskitia Project of PNUD/GEF and the UNAH, contribute with scientific information that could serve as tools for the Miskito community as to the management of their fishing resources.

Keywords: lagoon system, artisanal fisheries, sustainable handling, fishery resources, exploitation, total mortality. 


\section{INTRODUCCIÓN}

En la costa caribeña de la Moskitia hondureña hay un sistema amplio y complejo de lagunas, cubriendo un área total de alrededor de 80,000 hectáreas, cuyas aguas van desde agua fresca a agua salobre. Las lagunas están conectadas por una gran cantidad de riachuelos, que típicamente están rodeados por manglares (Prodoc Proyecto $\left.N^{\circ} 70863,2009\right)$. Este complejo de lagunas proporciona grandes poblaciones de peces que se reproducen y maduran en riachuelos y en ensenadas del área, sosteniendo la pesca identificada como el principal medio de vida para las comunidades Miskitas que habitan el Sistema Lagunar de Karataska (Prodoc Proyecto $N^{\circ} 70863$, 2009).

Esta área de difícil acceso en transporte terrestre, conserva su integridad y biodiversidad proporcionando servicios ecosistémicos como la pesca comercial industrial y artesanal (Prodoc Proyecto $\left.N^{\circ} 70863,2009\right)$. La pesca industrial genera empleo en las comunidades miskitas, desempeñándose generalmente como buzos y marineros para la captura de la langosta espinosa (Panulirus argus), caracol (Strombus gigas) y recientemente de pepino de mar (Holothuria mexicana / Sostichopus badionatus) (Prodoc Proyecto $\mathrm{N}^{\circ} 70863,2009$ ). Desde el 2010 , se suma a las actividades artesanales la pesca de medusa de mar (Stomolophus meleagris) (Ortiz-Lobo et al. 2014).

Para la zona de la Moskitia hondureña se registran 69 especies de peces distribuidas en los diferentes cuerpos de agua dulce, salobre y salada (Matamoros et al. 2009); de las cuales se benefician cerca de 80 mil personas en su mayoría del pueblo indígena Miskito (Matamoros et al. 2009). Para la elaboración del plan de manejo pesquero para el Sistema Lagunar de Karataska, Carrasco y Colindres (2012) registran 80 especies distribuidas entre peces, moluscos y crustáceos; el $40 \%$ de estas especies son de interés económico y cultural. Las comunidades indígenas miskitas del Sistema Lagunar de Karataska se dedican mayoritariamente durante los meses de septiembre a marzo de la pesca artesanal tanto para el autoconsumo como para la venta a pequeña escala de robalo y otras especies (pescado seco salado) (López, 2013). No tienen mayor comercialización debido a la falta de condiciones de infraestructura para su acopio y comercialización (López, 2013). El pescado seco salado se comercializa a San Pedro Sula y La Ceiba (López, 2013).

La pesca de robalo se ha convertido en una de las principales preocupaciones para los pescadores artesanales, debido a la reducción de capturas y tallas menores de los peces, siendo el esfuerzo de pesca mayor (Carrasco y Colindres, 2012). A lo ante- 
rior también se suma la demanda de la población de pescadores que se integrarán a la pesca artesanal del robalo, con el cierre de la pesca de langosta por buceo (SAG, 2011; Carrasco y Colindres, 2012), actividad en la que se ocupan al menos unos cuatro mil buzos por temporada (Carrasco y Colindres, 2012). Para alcanzar el manejo sostenible de los recursos pesqueros tradicionales del robalo y la kawacha en la laguna de Karataska, el Proyecto Moskitia de PNUD/GEF con la asesoría de la UNAH, evaluó las poblaciones de especies ícticas de interés cultural y económico del Sistema Lagunar de Karataska. Esta evaluación comprendió el análisis estadístico de los datos de biometrías, estimación de la mortalidad total (Z) y el cálculo preliminar del rendimiento máximo sostenible (RMS) de una especie de robalo (Centropomidae undecimalis) y kawacha (Eugerres plumieri) capturadas en el sistema lagunar en el periodo 2012-2014.

De esta manera el Proyecto Moskitia de PNUD/GEF y la UNAH aportan conocimientos científicos, con el fin de brindar herramientas de manejo a los pobladores miskitos para el ordenamiento de sus recursos pesqueros. El objetivo de este trabajo fue evaluar el estado de la pesquería de dos especies de interés cultural y económico que son fuente de ingresos económicos y alimento en el Sistema Lagunar de Karataska.

\section{MATERIALES Y MÉTODO}

El presente estudio contó de dos fases: la primera consistió en la aplicación de los principios de ciencia ciudadana (Hoffman, 2016), que se basan en la participación de voluntarios de la comunidad en general en investigación académica para abordar problemas del mundo real (Wiggins y Crowston, 2011; Tulloch, 2015). Como cualquier otro tipo de investigación, la ciencia ciudadana presenta limitaciones y sesgos que hay que considerar y controlar (Hoffman, 2016), por lo cual, se contrataron y capacitaron nueve pescadores locales como para-técnicos (Figura 1), sus actividades consistieron en la identificación taxonómica y toma de datos biométricos de las especies de interés comercial de las comunidades miskitas muestreadas.

La segunda fase del estudio se realizó con aplicación de modelo analítico en las áreas de desembarque pesquero de las comunidades miskitas de: Aurata, Yahurabila, Palkaka, Prumnitara, Uhí y Uhí Landing, estas dos últimas se encuentran colindantes y su separación se debe a la zona de pesca, Uhì faena en el mar y Uhí Landing en la laguna de Karataska, estas comunidades pertenecen a la jurisdicción del Muni- 
cipio de Puerto Lempira $\left(15^{\circ} 15^{\prime} 55^{\prime \prime} \mathrm{N}\right.$ y $83^{\circ} 46^{\prime} 28^{\prime \prime}$ W) y Cruta o Walpatara, única

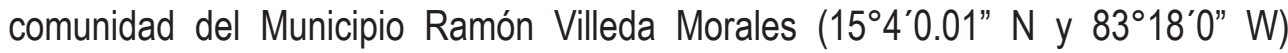
tomada en consideración para el estudio, debido a su alta población dedicada a la pesca. Estas comunidades están situadas en la periferia de la Laguna de Karataska, exceptuando la comunidad de Cruta, ya que se encuentra en las cercanías de la desembocadura del río Cruta (Figura 2).

Figura 1. Primera fase de estudio: capacitación de pescadores locales como paratécnicos en la toma de datos biométricos e identificación taxonómica de especies ícticas en Puerto Lempira, Gracias a Dios, 2012.

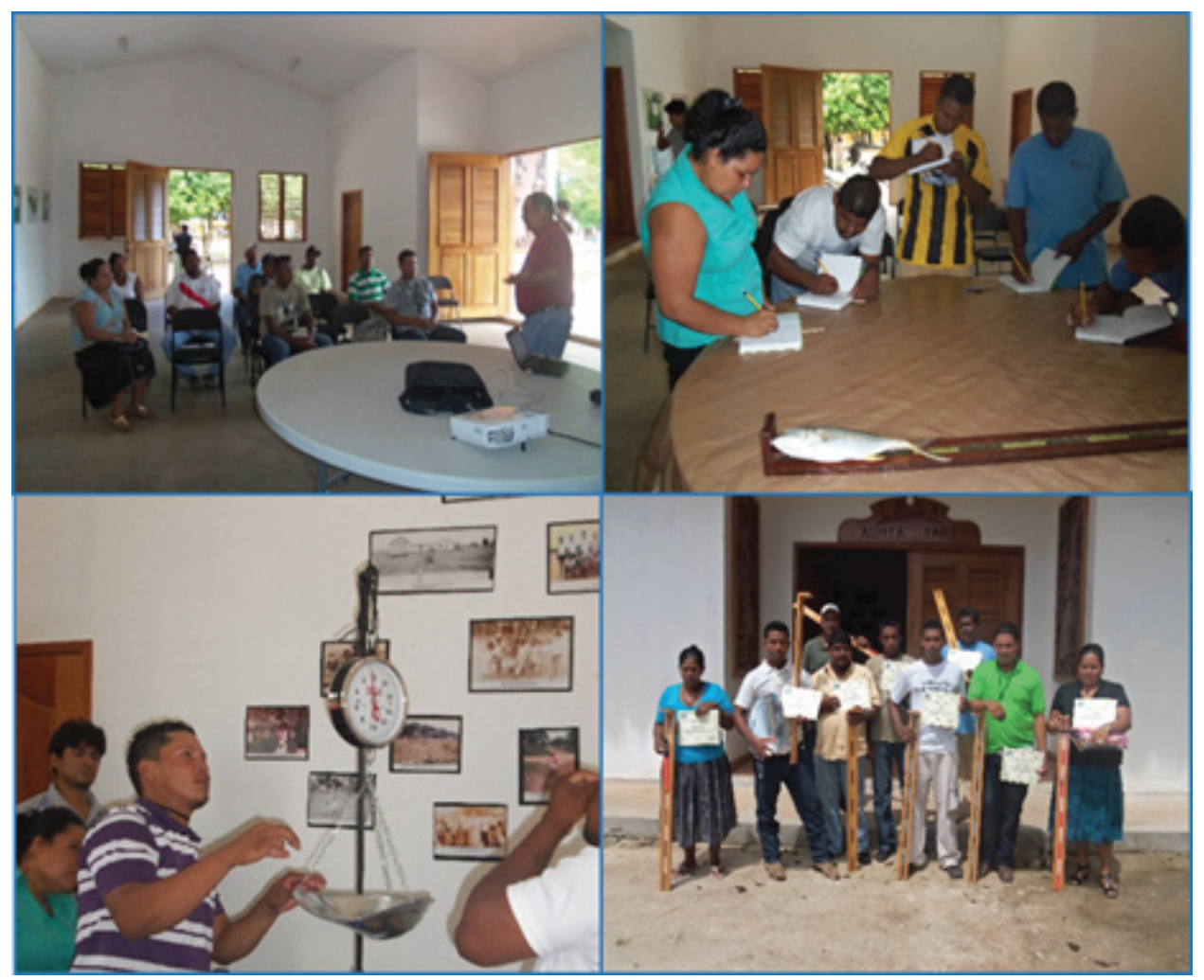

De octubre de 2012 hasta septiembre de 2014 los para-técnicos hicieron muestreos al azar, cuando los pescadores regresaban de su jornada de pesca. La toma de muestras se realizó para embarcaciones locales a lo largo de los meses; aunque no se revisaron todas las embarcaciones en cada visita, se midieron todos los ejemplares capturados por las embarcaciones seleccionadas en cada muestreo. A cada espécimen se le tomaron los datos de: nombre común (nombre coloquial por los pescadores), nombre científico, longitud total en $\mathrm{cm}(\mathrm{LT})$, con un ictinómetro de $1.10 \mathrm{~m}$ 
de longitud máxima, con una lectura mínima a $\pm 1 \mathrm{~mm}$, y el peso sin evisceración en onzas (oz) con una balanza mecánica de reloj con capacidad de 1oz \pm 40 libras, además se determinó el sexo y el estadio gonadal (Berthou, et al. 2001).

Figura 2. Áreas de desembarque pesquero de las comunidades miskitas consideradas para evaluar las especies de interés cultural y económico del Sistema Lagunar de Karataska, Gracias a Dios, 2012-2014.

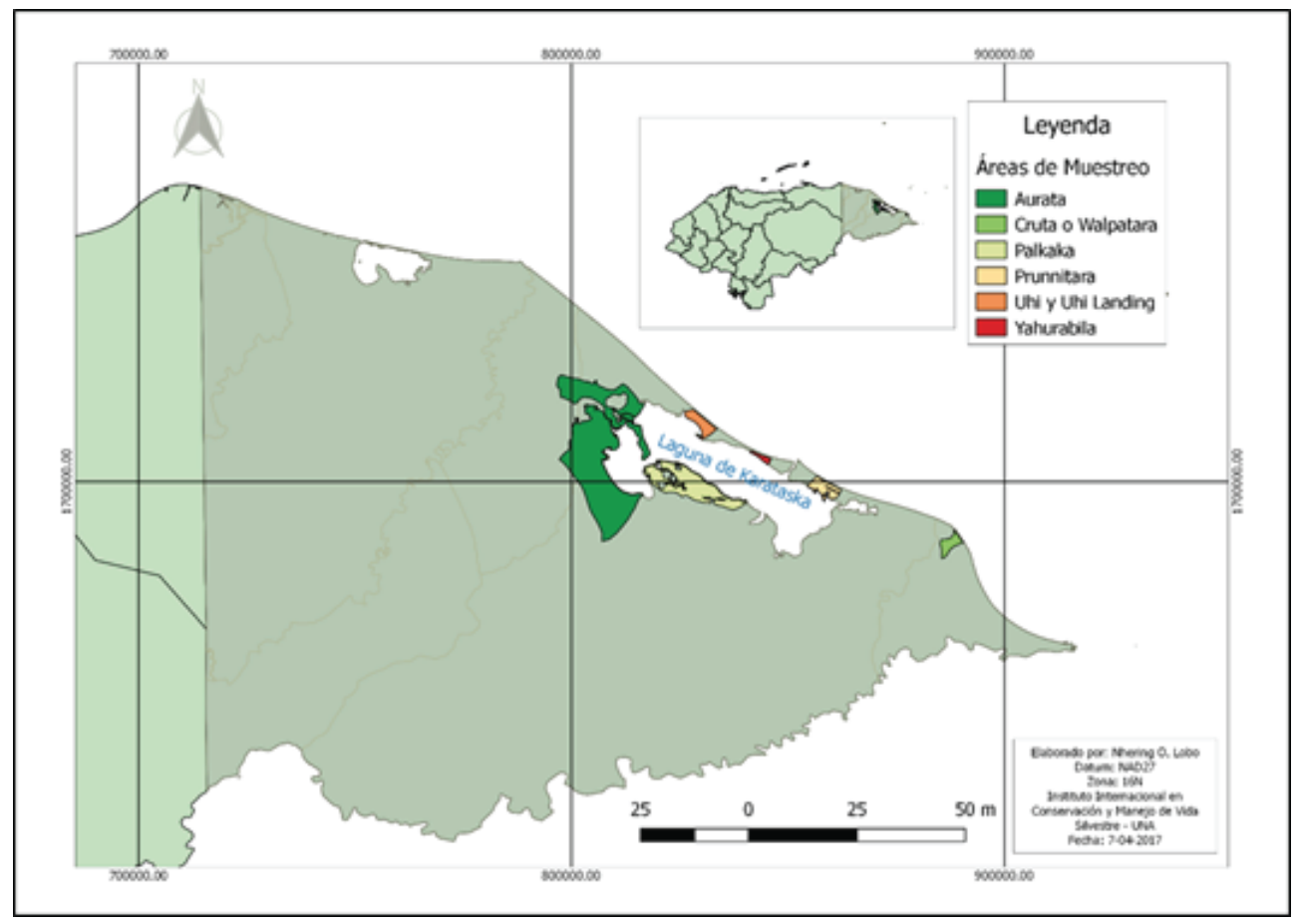

\section{Análisis estadístico}

La finalidad básica de la evaluación de los stocks pesqueros es la búsqueda del nivel de explotación que permita obtener, a largo plazo, el rendimiento máximo sostenible en peso de una pesquería (Sparre y Venema, 1997). Ante la ausencia de una secuencia de estructuras de tallas históricas de los desembarques, para este estudio sobre el estado de la pesquería en el Sistema Lagunar de Karataska, se evaluó el stock presente durante la temporada 2012-2014 basándose en el modelo "análisis de pseudo-cohorte a la talla" (Jones 1984).

El método analítico basado en la dinámica de poblaciones, utiliza la distribución de frecuencias de tallas de longitud total (LT) de los organismos (Sparre y Venema, 1997). Este método consiste en la estimación de la mortalidad total (Z) a partir de la 
curva de captura linealizada para el análisis de rendimiento máximo sostenible (RMS) por medio del análisis de cohorte de Jones (Sparre y Venema, 1997).

Para elaborar la curva de captura linealizada convertida a tallas, la cual se utilizó para estimar la Mortalidad Total (Z), se empleó la siguiente formula:

$$
\frac{\ln \mathrm{C}(\mathrm{L} 1 . \mathrm{L} 2)}{\Delta \mathrm{t}(\mathrm{L} 1, \mathrm{~L} 2)}=\mathrm{c}-\mathrm{Z}^{*} \mathrm{t} \frac{\mathrm{L} 1+\mathrm{l} 2}{2}
$$

Donde In C (L1.L2) logaritmo natural de la captura, $\Delta \mathrm{t}$ (L1.L2) es el tiempo que tarda el organismo en crecer de la talla 1 (L1) a la talla 2 (L2). Este es una ecuación lineal donde la pendiente equivale a la mortalidad total (Sparre y Venema, 1997). De igual modo, se estimó la talla media de captura (TMC) y la talla media de madurez sexual (TMM).

Para estimar la Mortalidad por Pesca, se despejo para el valor F utilizando la ecuación de captura basada en tallas, la que corresponde a la siguiente función:

$$
\mathrm{C}(\mathrm{L} 1, \mathrm{~L} 2)=\mathrm{N}(\mathrm{L} 1)^{*} \mathrm{~F} / \mathrm{Z}^{*}\left[1-\exp \left(-\mathrm{Z}^{*} \Delta \mathrm{t}\right)\right]
$$

Donde C(L1,L2) número de peces que son capturados entre las longitudes L1 y L2; $\mathrm{N}(\mathrm{L} 1)$ número de peces que alcanzan la longitud L1 (también llamados como el número de sobrevivientes); F mortalidad por pesca; Z mortalidad total; $\Delta \mathrm{t}$ tiempo que tarda el organismo en crecer de la talla 1 (L1) a la talla 2 (L2).

- Finalmente, para el análisis del rendimiento máximo sostenible (RMS) por medio del análisis de cohorte de Jones, basado en las tallas (Sparre y Venema, 1997), se determinó con la siguiente expresión:

$$
\operatorname{Exp}\left(\frac{M}{2} * \Delta t\right)=\exp \left(\frac{M}{2} * \frac{1}{K} * \operatorname{In}\left(\frac{\mathrm{L} \infty-\mathrm{L} 1}{\mathrm{~L} \infty-\mathrm{L} 2}\right)\right)
$$

\section{RESULTADOS}

\section{Composición Ictiofaunística:}

Se midieron y pesaron un total de 7085 peces, identificándose 26 especies de interés pesquero en el Sistema Lagunar de Karataska pertenecientes 15 familias, 21 géneros y 26 especies (Cuadro 1). Las familias con mayor cantidad de especies son: Centropomidae $(n=4,39 \%)$, Cichlidae $(n=4,10 \%)$ y Sciaenidae $(n=3.9 \%)$. Por otra parte, las 
especies más abundantes fueron: Centropomus undecimalis ( $n=1398,20 \%)$, Eugerres plumieri $(n=1146,16 \%)$, Bagre marinus ( $n=711,10 \%)$, Centropomus pectinatus $(n=607,9 \%)$, Centropomus parallelus $(n=598,8 \%)$.

\section{Cuadro 1. Listado sistemático de las especies de peces del Sistema Lagunar de Kara- taska, Gracias a Dios, 2012-2014.}

\begin{tabular}{|c|c|}
\hline Familias & Especies \\
\hline Sphyrnidae & Sphyrna lewini (E. Griffith y C. H. Smith, 1834) \\
\hline Carcharhinidae & Rhizoprionodon porosus (Poey, 1861) \\
\hline Megalopidae & Megalops atlanticus (Valenciennes, 1847) \\
\hline Ariidae & Bagre marinus (Mitchill, 1815) \\
\hline Heptapteridae & Rhamdia guatemalensis (Günther, 1864) \\
\hline Mugilidae & Joturus pichardi (Poey, 1860) \\
\hline Mugilidae & Mugil cephalus (Linnaeus, 1758) \\
\hline Centropomidae & Centropomus ensiferus (Poey, 1860) \\
\hline Centropomidae & Centropomus parallelus (Poey, 1860) \\
\hline Centropomidae & Centropomus pectinatus (Poey, 1860) \\
\hline Centropomidae & Centropomus undecimalis (Bloch, 1792) \\
\hline Carangidae & Caranx hippos (Linnaeus, 1766) \\
\hline Carangidae & Oligoplites palometa (Cuvier, 1832) \\
\hline Lutjanidae & Lutjanus griseus (Linnaeus, 1758) \\
\hline Lutjanidae & Lutjanus jocu (Bloch y Schneider, 1801) \\
\hline Gerreidae & Eugerres plumieri (Cuvier, 1830) \\
\hline Sparidae & *Vieja maculicauda (Regan, 1905) \\
\hline Sciaenidae & Sphyraena guachancho (Cuvier, 1829) \\
\hline Sciaenidae & Cynoscion virescens (Cuvier, 1830) \\
\hline Sciaenidae & Bairdiella ronchus (Cuvier, 1830) \\
\hline Cichlidae & Menticirrhus littoralis (Holbrook, 1847) \\
\hline Cichlidae & Oreochromis niloticus (Linnaeus, 1758) \\
\hline Cichlidae & Parachromis dovii (Günther, 1864) \\
\hline Cichlidae & Sphyraenidae \\
\hline Scombridae & Scomberomorus brasiliensis (Collette, Russo y Zavala-Camin, 1978) \\
\hline
\end{tabular}

Nota: para este estudio se reportará a la especie Vieja maculicauda sin considerar su cambio taxonómico a Paraneetroplus maculicauda, ya que su modificación fue en años posteriores al estudio (Mcmahan, C. D., Matamoros, W. A., Piller, K. R., y Chakrabarty, P. 2015). 
El listado contiene una especie exótica (Oreochromis niloticus), introducida a Honduras con fines de acuacultura desde la década de los cincuentas (FAO, 2008). Las localidades que mostraron mayor riqueza y abundancia relativa fueron: Uhí $(n=22$, $20 \%)$, Aurata $(n=21,19 \%)$, Prumnitara $(n=18,20 \%)$. Sin embargo, la comunidad de Cruta presentó una alta abundancia con una baja riqueza de especies $(n=14,21 \%)$. Las comunidades de Yahurabila $(n=10,1 \%)$ y Palkaka $(n=12,2 \%)$ reportaron la menor cantidad de especies durante el estudio (Figura 3), debido a que los para-técnicos tuvieron que retirarse con anticipación, ya que no se dedicaban exclusivamente a la pesca.

Figura 3. Riqueza y abundancia de la composición ictiofaunística de las comunidades pesqueras miskitas del Sistema Lagunar de Karataska, Gracias a Dios, 2012-2014. Abreviaturas: AUR: Aurata, KRU: Cruta, PAL: Palkaka, PRU: Prumnitara, UHI: Uhí, UHI_LAN: Uhí Landing y YAH: Yahurabila.

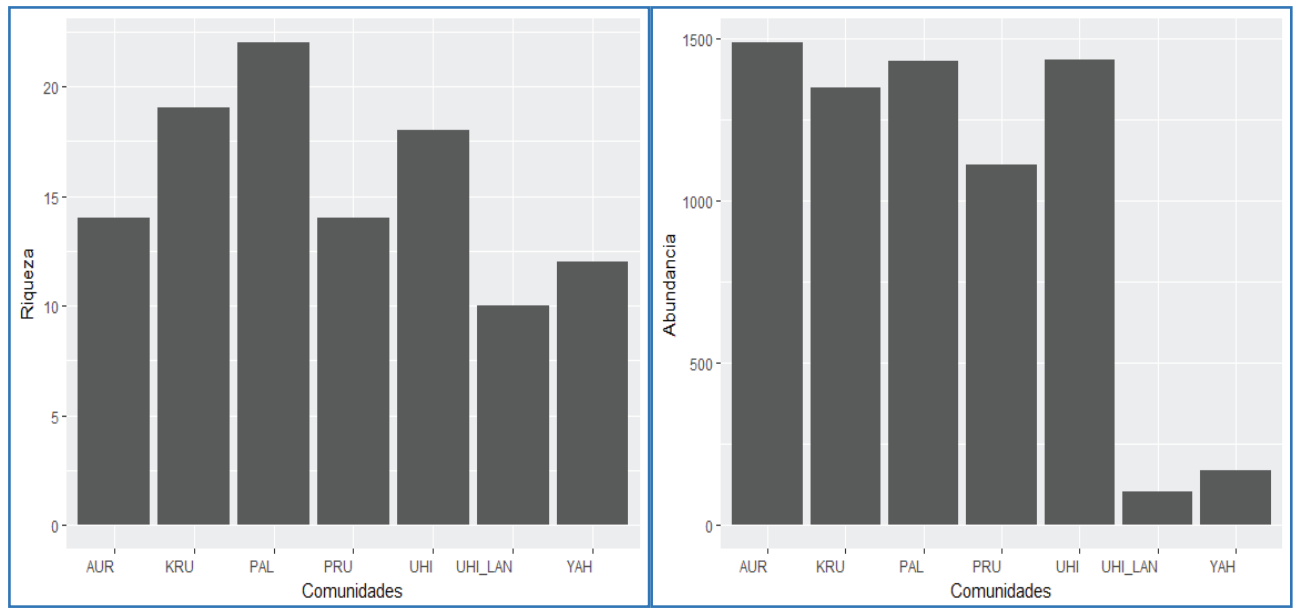

\section{Análisis de cohorte de Jones}

Se trabajó la distribución de frecuencias de tallas con cuatro especies dominantes en el área de estudio, por su abundancia y distribución; asimismo consideradas especies ícticas capturadas para el autoconsumo o con perspectivas comerciales en la mayoría de las familias en la Moskitia (MASTA 2012). Hecha esta salvedad, se estimó para Centropomus undecimalis, Eugerres plumieri, Centropomus pectinatus y Centropomus parallelus la talla media de captura (TMC) y la talla media de madurez sexual (TMM) (Figura 4). No obstante, para el análisis de cohorte de Jones solo se utilizó los datos de Centropomus undecimalis y Eugerres plumieri, debido a su considerable abundancia de datos e interés culturar. 
Figura 4. Distribución de frecuencias de tallas de las cuatro especies de interés comercial y cultural en el Sistema Lagunar de Karataska, Gracias a Dios, 2012-2014.

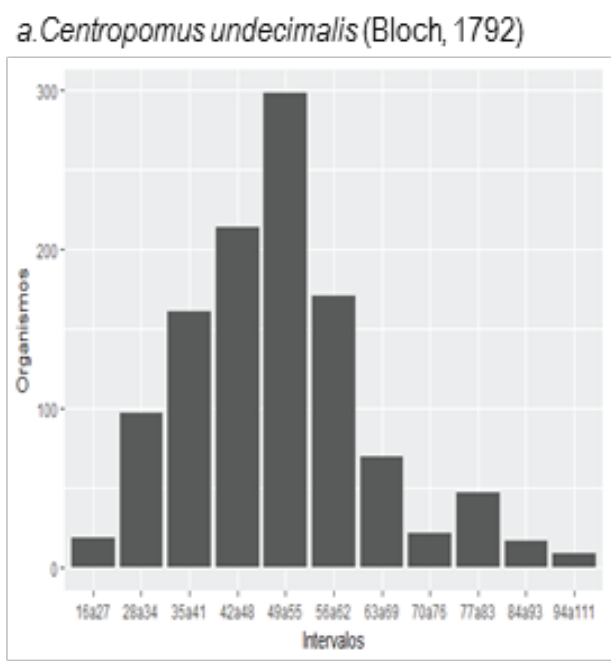

c. Centropomus pectinatus (Poey, 1860)

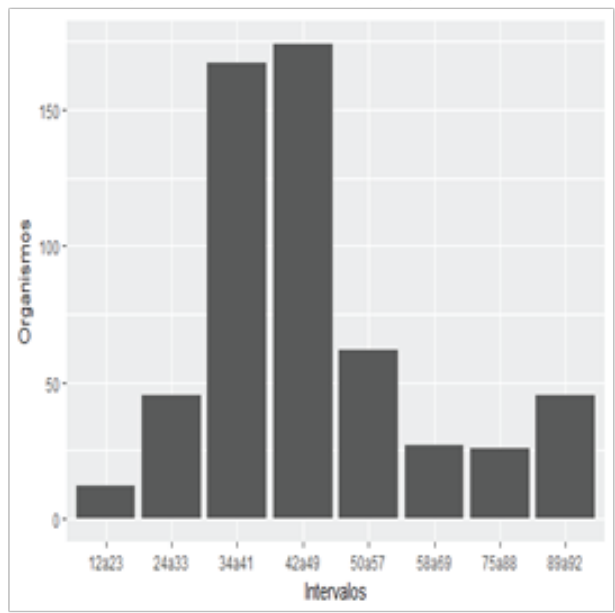

b. Eugerres plumieri (Cuvier, 1830)

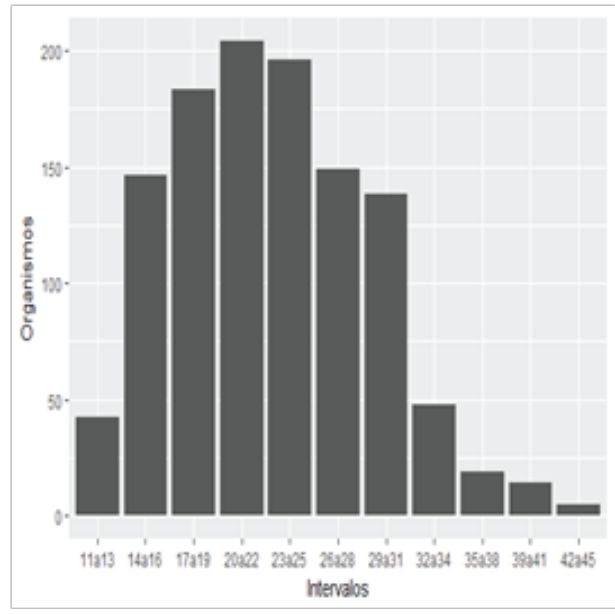

d. Centropomus parallelus (Poey, 1860)

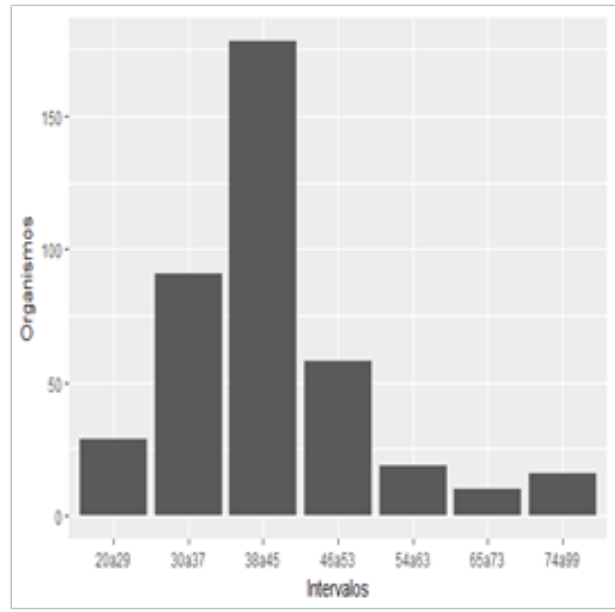

Centropomus undecimalis (Figura 5a). La talla media de captura es de $50.63 \mathrm{~cm}$, en cambio la talla media de maduración sexual (L50) para las Hembras es de $48.76 \mathrm{~cm}$ y para Machos es de $48.76 \mathrm{~cm}$. Sin embargo, la talla de primera captura está dentro del rango de $16-27 \mathrm{~cm}$ (Figura 4.a). La talla máxima de captura fue reportada en la comunidad de Palkaka con un espécimen de $111 \mathrm{~cm}$ LT y 11,804 gr. 
Eugerres plumieri (Figura $5 \mathrm{~b}$ ). La talla media de captura es de $22.83 \mathrm{~cm}$, por otra parte, la talla media de maduración sexual (L50) para las hembras es de $22.70 \mathrm{~cm}$ y para Machos es de $24.65 \mathrm{~cm}$. No obstante, la talla de primera captura está dentro del rango de $11-13 \mathrm{~cm}$ (Figura 4.b). La talla máxima de captura se reportó en la comunidad de Prumnitara con un espécimen de 45cm LT y 1,475.5 gr.

Centropomus pectinatus (Figura 5c). La talla media de captura es de $48.15 \mathrm{~cm}$, por el contrario, la talla media de maduración sexual (L50) para las hembras es de $49 \mathrm{~cm}$ y para los Machos es de $42 \mathrm{~cm}$. En cambio, la talla de primera captura está dentro del rango de $12-23 \mathrm{~cm}$ (Figura 4.c). La talla máxima de captura se reportó en las comunidades de Kruta y Palkaka, con seis y un espécimen respectivamente con $92 \mathrm{~cm} \mathrm{LT,} \mathrm{y}$ presentando un espécimen de Kruta el mayor peso de 6,810 gr.

Centropomus parallelus (Figura $5 \mathrm{~d}$ ). La talla media de captura es de $42.46 \mathrm{~cm}$, aunque la talla media de maduración sexual (L50) para las hembras es de $40 \mathrm{~cm}$ y para los machos es de $41 \mathrm{~cm}$. Sin embargo, la talla de primera captura está dentro del rango de $20-29 \mathrm{~cm}$ (Figura 4.d). La talla máxima de captura se reportó en la comunidad de Kruta con un espécimen de 99cm LT y 8,626 gr.

Figura 5. Imágenes de las cuatro especies de interés comercial y cultural en el Sistema Lagunar de Karataska, Gracias a Dios, 2012-2014.

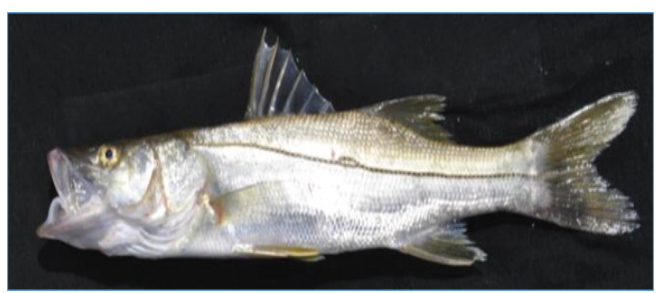

Figura 5a. Centropomus undecimalis.

Fuente: Fotografía de Carrasco (2014).

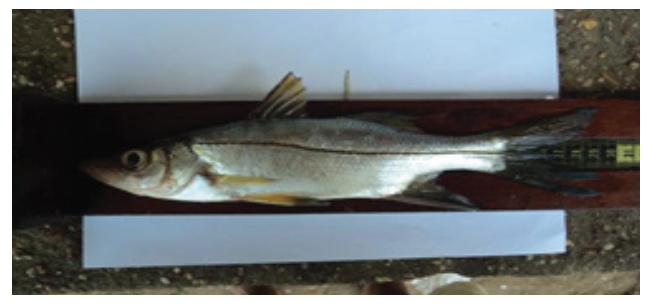

Figura 5c. Centropomus pectinatus.

Fuente: Fotografía de José Luis Andrade,

Prumnitara- Fishbase.

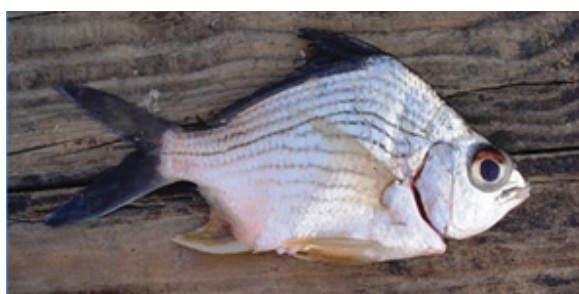

Figura 5b. Eugerres plumieri.

Fuente: Fotografía de Trevor Meyer - Fishbase

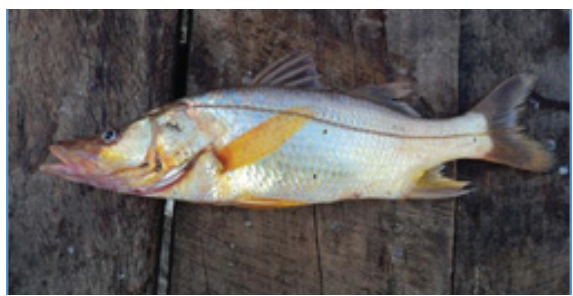

Figura 5d. Centropomus parallelus.

Fuente: Fotografía de José Luis Andrade,

Barra del Río Cruta - Fishbase. 
La Mortalidad Total (Z) se estimó con la curva de captura linealizada basada en tallas y la utilización de las constantes recomendadas por la bibliografía próxima a la región (Figura 6 y 7). Para Centropomus undecimalis: $K=0.145$ por año, $L \infty=143 \mathrm{~cm}$ (Caballero-Chávez, V. 2009), y Eugerres plumieri: K:0.78 por año, L $\infty=46 \mathrm{~cm}$ (Olaya-Nieto, C.W., y Appeldoorn, R. S. 2004).

Figuras 6 y 7. Mortalidad Total (Z) y Mortalidad por Pesca (F) para Centropomus undecimalis en el Sistema Lagunar de Karataska, Gracias a Dios, 2012-2014.

6)

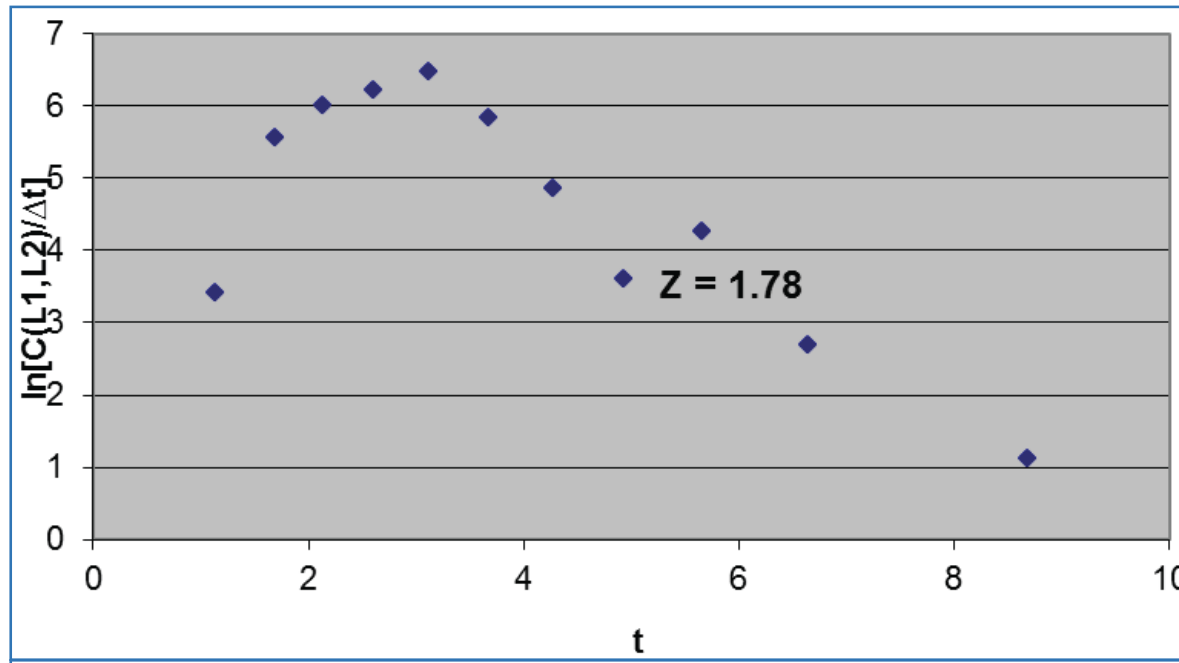

7)

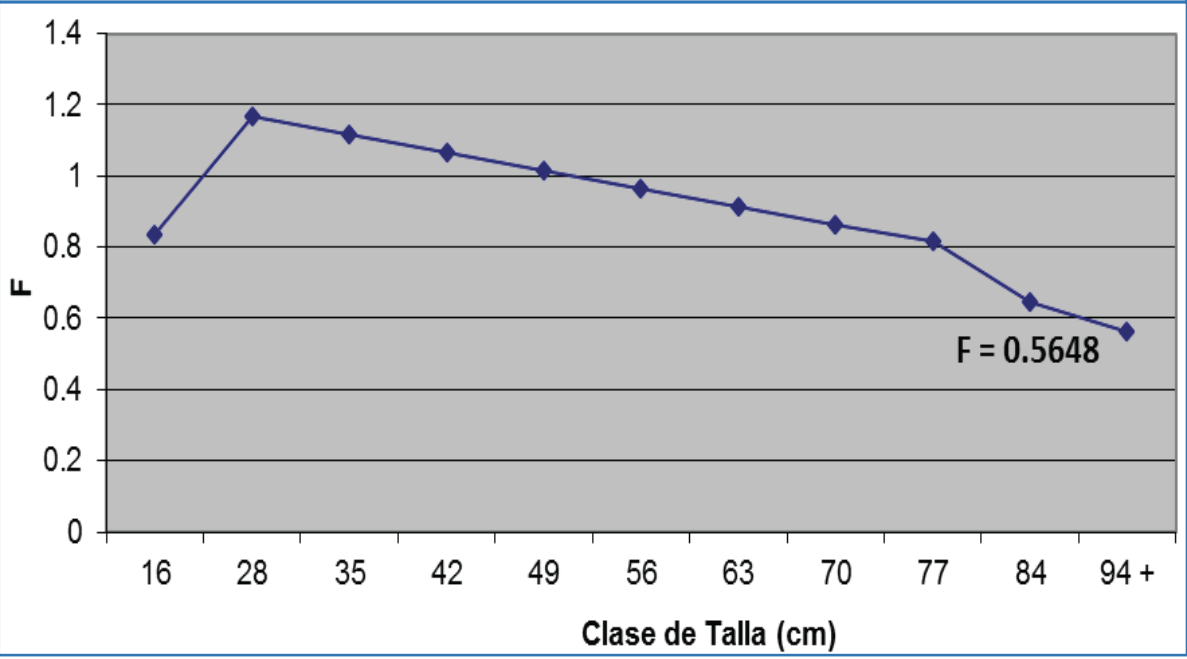


Atendiendo la información de organismos adultos de 29 a $31 \mathrm{~cm}$ de LT de Eugerres plumieri, la estimación de la Mortalidad Total fue Z=1.67 (límite de confianza de $95 \%$ ) (Fig. 8), y la Mortalidad por Pesca $F=0.63$ (Fig. 9). El resultado del análisis de rendimiento por recluta para organismos adultos fue de RMS $=178604.59$ toneladas.

Figuras 8 y 9. Mortalidad Total (Z) y Mortalidad por Pesca (F) para Eugerres plumieri en el Sistema Lagunar de Karataska, Gracias a Dios, 2012-2014.

8)

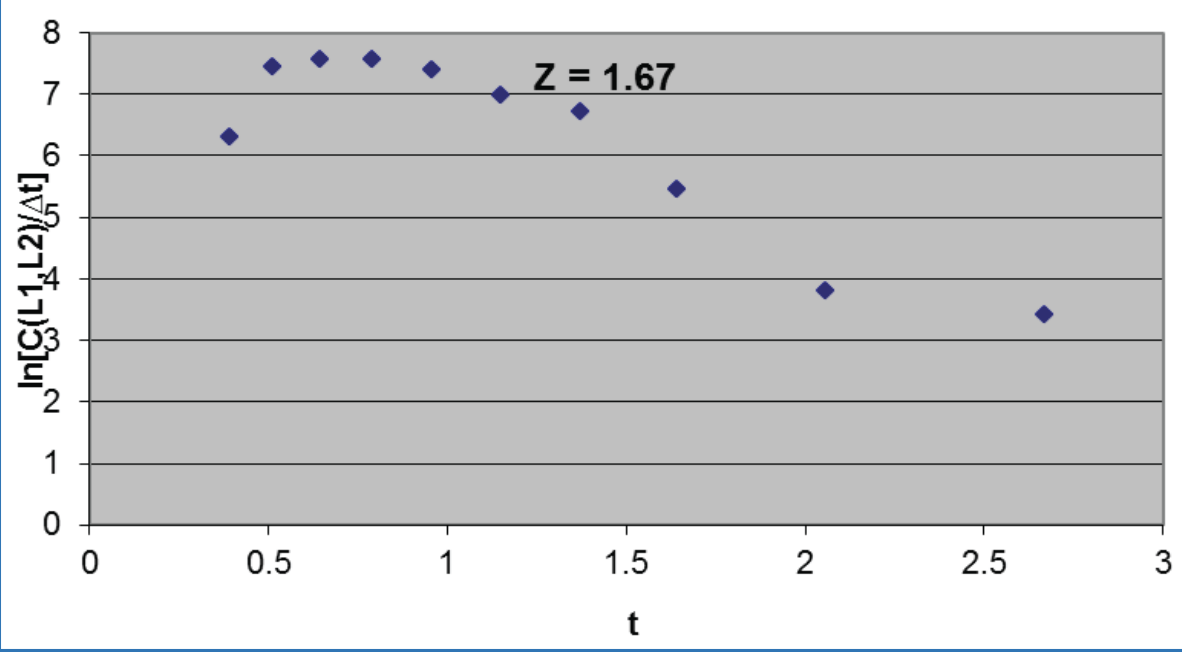

9)

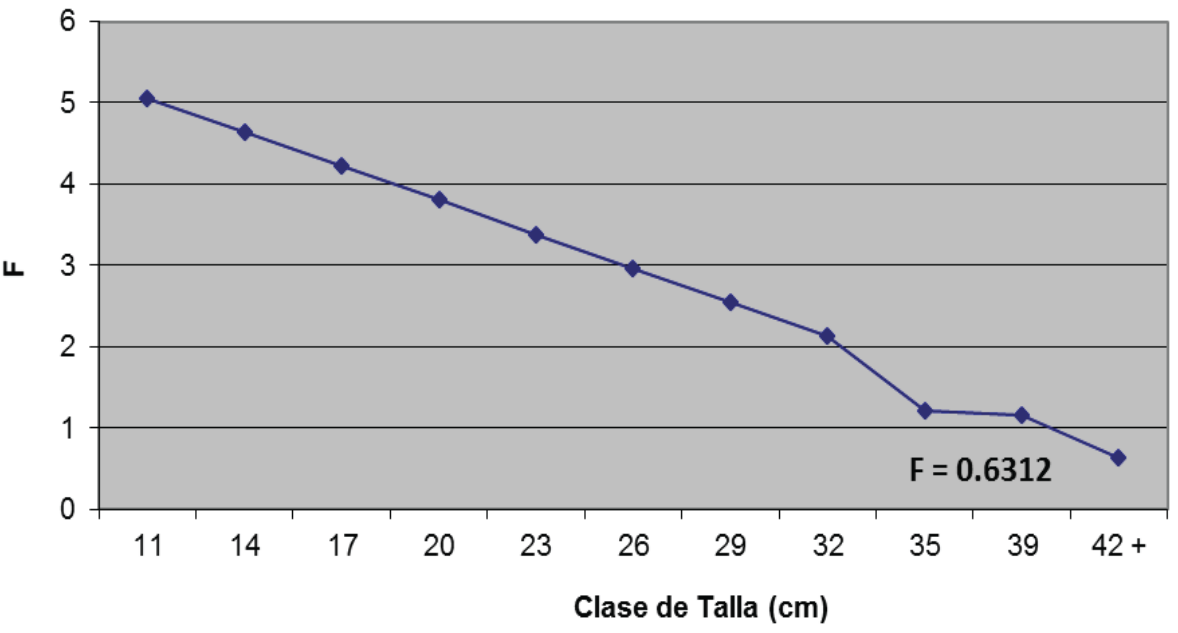




\section{DISCUSIÓN}

La riqueza reportada en este estudio fue menor que la obtenida por Carrasco (2014), reportando 89 especies de peces pertenecientes a 37 familias. Este autor consideró que al menos 50 especies son de consumo frecuente en las comunidades locales; no obstante, para este estudio se especifican especies de interés pesquero. Carrasco (2014) y en este trabajo se reporta una especie exótica la tilapia (Oreochromis niloticus).

Los resultados obtenidos en la distribución de frecuencia de tallas, muestran que para tres especies de robalo (Centropomus undecimalis, C. pectinatus y C. parallelus) la talla media de captura supera la talla de madurez sexual (Andrade-Campos, et al. 2015). Sin embargo, para la C. undecimalis la mayoría de los individuos capturados, no llegan a la talla de primera maduración.

Por otra parte, el reclutamiento (ingreso a la pesquería) para las tres especies de robalo (Centropomus undecimalis, $C$. pectinatus y $C$. parallelus) se encuentra entre los 16,12 y $20 \mathrm{~cm}$, lo que puede comprometer el reclutamiento de las próximas generaciones al capturar juveniles. Esta situación puede corregirse aumentando la luz de malla de las redes de pesca.

En el caso de la kawacha (Eugerres plumieri) la talla media de captura coincide con la talla media de madurez sexual en hembras y no supera la de los machos (Andrade-Campos, et al. 2015), lo que indica el riesgo de captura de juveniles; el reclutamiento inicia a los $11 \mathrm{~cm}$ (Andrade-Campos, et al. 2015), lo que pone en riesgo el potencial reproductor y abastecimiento de individuos a futuro para la pesquería.

En la estimación de la mortalidad total se puede observar que las especies de Centropomus undecimalis y Eugerres plumieri, se encuentra en fases de explotación intensa, 1.78 y 1.67 respectivamente, lo que requiere de medidas de ordenamiento. En Campeche, Golfo de México se encontró una mortalidad total $(Z)$ de 1.15 para la misma especie (Caballero, 2012).

Por medio del análisis de cohorte de Jones, se obtuvo de manera muy preliminar el rendimiento máximo sostenible (RMS) para Centropomus undecimalis de 69193.17 toneladas y para Eugerres plumieri, de 178604.59 toneladas. Sin embargo, se debe considerar que esta es una pesquería con pocos datos (dos años) y no se cuenta con un sistema estatal de estadistas de producciones totales de la zona que permita 
comparar estos rendimientos, con el fin determinar si se sobrepasa o no la pesca sostenible.

\section{CONCLUSIONES}

La riqueza presente en el sistema lagunar de Karataska demuestra porque es considerado el más grande, mejor conservado y más biodiverso de los humedales de la región Centroamericana (Carrasco y Flores 2008). Sin embargo, se exhorta a continuar con el monitoreo de especie de interés comercial, consumo y exóticas para garantizar la seguridad alimentaria de la zona.

Para reducir la presión pesquera se debe considerar modificar la selectividad de las artes de pesca con el aumento de luz de malla a cuatro pulgadas, evitando con esta medida a futuro, la captura de juveniles. También se debe reducir el esfuerzo de pesca con redes para disminuir la tasa de explotación.

Se recomienda la explotación de un $40 \%$ del rendimiento máximo sostenible (RMS) obtenido de 69193.17 toneladas para Centropomus undecimalis y un $50 \%$ del rendimiento máximo sostenible (RMS) obtenido de 178604.59 toneladas para Eugerres plumieri. Lo anterior, basándose en los regímenes de explotación intensa encontrados y en la aplicación del enfoque precautorio de la FAO para la sostenibilidad del recurso pesquero. Ante la ausencia de un sistema de estadística pesquera en la zona, se recomienda robustecer este análisis con un sistema de monitoreo permanente.

\section{AGRADECIMIENTOS}

A todos los pescadores artesanales miskitos, y para-técnicos quienes fueron los encargados de colectar los datos desde octubre del 2012 hasta septiembre de 2014, durante las temporadas de pesca. A PAMUPEL y MASTA, por apoyar en la selección de los para-técnicos, participar durante las visitas para socializar el proceso de colecta de datos, y demostrar apertura para trabajar en territorio Miskitu; y especialmente a los Bachilleres Técnicos en Computación, Lee Toribio Trapp, Emilia Janeth Torres Ávila y Karla Selene Avilés, quienes se encargaron de tabular los datos colectados. A Elina Kaartinen Coordinadora Nacional del Proyecto Moskitia. 


\section{REFERENCIAS BIBLIOGRÁFICAS}

(2009). Prodoc Proyecto N 70863. Proyecto Conservación de la Biodiversidad en los paisajes productivos indigenas de la Moskitia. Tegucigalpa.

Andrade-Campos, J. (2012). Fotografía de Centropomus parallelus. Barra del Rio Kruta, Gracias a Dios - Honduras. 07-04-2017, de fishbase Sitio web: http://www.fishbase.or$\mathrm{g} /$ photos/UploadedBy.php?autoctr=17842\&win=uploaded.

Andrade-Campos, J. (2012). Fotografía de Centropomus pectinatus. Prumnitara, Puerto

Lempira, Gracias a Dios - Honduras. 07-04-2017, de fishbase Sitio web: http://www.fishbase.org/photos/UploadedBy.php?autoctr=17904\&win=uploaded.

Andrade-Campos, J.L., Ortiz-Lobo, N.D., y Agar-Lopez, E. (2015). Informe: Evaluación pesquera de cuatro especies de interés cultural y económico en el Sistema Lagunar de Karataska. Proyecto Conservación de la Biodiversidad en Paisajes Productivos Indígenas de la Mosquitia hondureña, PNUD/GEF. Tegucigalpa, M.D.C. 37p.

Aríz Abarca, L., Jerez Aranda, G. E., Miranda Pérez, H. H., y Céspedes, I. (1996). Informe final. Análisis e investigación del stock del recurso macha (Mesodesma donacium) en Putu y La Trinchera, VII región. Instituto de Fomento Pesquero (IFOP). Chile.

Berthou, P., Oquelí, M. D., López, E., Gobert, B., Macabiau, C., y Lespagnol, P. (2001).

Diagnóstico de la pesca artesanal de las Islas de la Bahía, Proyecto Manejo Ambiental de las Islas de la Bahía.

Caballero-Chávez, V. (2009). Pesquería de robalo blanco Centropomus undecimalis en Campeche. Ciencia Pesquera, 17(2), 77-86.

Caballero-Chávez, V. (2012). Evaluación de la Pesquería de robalo blanco Centropomus undecimalis en Ciudad del Carmen, Campeche. Ciencia Pesquera, 20(2), 35-42.

Carrasco, J. C. (2014). Monitoreo Ecológico del Sistema Lagunar Karataska: Énfasis en ecología de comunidad de peces. Programa de las Naciones Unidas para el Desarrollo. Puerto Lempira: Proyecto Conservación de La Biodiversidad en los Paisajes Productivos Indígenas. INCEBio. Doc. Tec. $18 \mathrm{p}$.

Carrasco, J. C., y Colindres, I. (2012). Plan de Manejo del Sistema Lagunar de Karataska. Programa de las Naciones Unidas para el Desarrollo. Puerto Lempira: Proyecto Conservación de La Biodiversidad en los Paisajes Productivos Indígenas.

Carrasco, J.C y Flores, R. 2008. Inventario de Humedales de la República de Honduras.

Secretaria de Recursos Naturales y Ambiente/USAID-MIRA. Honduras. 253pp.

FAO. (2008). Visión General del Sector Acuícola Nacional, Honduras. 8.

Jones, R. (1984). Assessing the effects of changes in exploitation pattern using length composition data (with notes on VPA and cohort analysis) (Vol. 256). Roma: FAO.

López, J.A. (2013). Guía Práctica sobre el Manejo, Manipulación, Transporte y Comercialización de Productos y Mariscos con Pescadores Artesanales. Programa Regional de USAID Para El Manejo De Recursos Acuáticos Y Alternativas Económicas.

MASTA (Mosquitia Asla Takanka-Unidad de la Mosquitia). (2012). Protocolo Bio-Cultural del Pueblo Indígena Miskita. UICN, MASTA. 
Matamoros, W. A., Schaefer, J. F., y Kreiser, B. R. (2009). Annonated checklist of the freshwater fishes of continental and insular Honduras. Zootaxa, 38.

Mcmahan, C. D., Matamoros, W. A., Piller, K. R., y Chakrabarty, P. (2015). Taxonomy and systematics of the herichthyins (Cichlidae: Tribe Heroini), with the description of eight new Middle American Genera. Zootaxa, 3999(2), 211-234.

Meyer, T. (2007). Fotografía de Eugerres plumieri. Bocas del Toro, Panamá. Recuperado de: http://www.fishbase.org/photos/UploadedBy.php?autoctr=13499\&win=uploaded.

Olaya-Nieto, C. W., y Appeldoorn, R. S. (2004). Edad y crecimiento de la mojarra rayada, Eugerres plumieri (Cuvier), en la Ciénaga Grande de Santa Marta, Colombia; Age and growth of striped mojarra, Eugerres plumieri (Cuvier), in the Cienaga Grande de Santa Marta, Columbia.

Ortiz-Lobo, N. Gonzalez-Florentino, B. y Agar-Lopez, E. (2014). Informe: Cuota preliminar para la pesca de medusa de mar (Stomolophus meleagris), en apego al principio de precautoriedad. Proyecto Conservación de la Biodiversidad en Paisajes Productivos Indígenas de la Mosquitia hondureña, PNUD/GEF. Tegucigalpa, M.D.C. 56p.

Pauly, D. (1983). Algunos métodos simples para la evaluación de recursos pesqueros tropicales. Organización de las Naciones Unidas para la Agricultura y la Alimentación. FAO. Doc. Tec. Pesca, (234):49 p.

Secretaría de Agricultura y Ganadería Honduras [SAG]. (2011). Memoria - Reunión Piquerías en Honduras. Tegucigalpa, Honduras. 6 de octubre, 2011. Recuperado de: http://www.globalfishalliance.org/pdfs/2011/MEMORIA_Reunion\%20FAO_6OCT2011_final.pdf

Sparre, P. y Venema S.C. (1997). Introducción a la Evaluación de Recursos Pesqueros Tropicales. Parte 1. Manual. FAO Documento Técnico de Pesca. Nº 306.1 Rev. 2: 420 pág. 48. 